\title{
DESCRIPTION DE LA LARVE \\ DE XENOPSYLLA CUNICULARIS SMIT, 1957 (SIPHONAPTERA, PULICIDAE).
}

\author{
H. LAUNAY*
}

RÉSUMÉ La larve de Xenopsylla cunicularis est décrite pour la première fois.

Sa morphologie est comparée à celle des autres Xenopsylla du groupe conformis et à celle d'un autre Pulicidae parasite du lapin de garenne : Spilopsyllus cuniculi.

\section{Desciption of the larva of Xenopsylla cunicularis Smit, 1957 (Siphonap- tera, Pulicidae).}

SUMMARY. The larva of Xenopsylla cunicularis is described for the first time.

Its morphology is compared with that of other flea larvae belonging to the Xenopsylla conformis group, and with the larva of an other Pulicidae parasitic on the European rabbit: Spilopsyllus cuniculi.

\section{Introduction}

Xenopsylla cunicularis Smit 1957 (Siphonaptera, Pulicidae) est une puce spécifique du lapin de garenne Oryctolagus cuniculus. Connue du Maroc et de la Péninsule ibérique elle a été mise en évidence également en France, dans le Sud-Ouest (Beaucournu et Launay, 1977). Au cours de son cycle annuel ses effectifs atteignent des fortes densités (Launay, 1980) ; elle peut donc, à l'intérieur de son aire de répartition, jouer un rôle important dans la propagation de la myxomatose.

Dans le cadre d'une action pluridisciplinaire concertée, en vue d'une approche d'une prophylaxie de la myxomatose, il nous a paru nécessaire d'étudier la répartition de $X$. cunicularis dans un premier temps et par conséquent de pouvoir la reconnaître dans nos prélèvements non seulement au stade adulte, mais aussi au stade larvaire.

* Insitut de Parasitologie de l'Ouest, avenue du Professeur Léon Bernard, F 35043 Rennes Cedex. Accepté le 25 mars I98I. 


\section{Techniques}

Le matériel utilisé pour cette étude a été obtenu à partir d'élevages, la souche étant originaire du Sud-Ouest de la France.

En vue du montage, les larves, tuées et conservées dans l'alcool à $70^{\circ}$, sont éclaircies pendant 2 heures dans une solution de Potasse à $20 \%$, à la température ambiante. Après rinçage et déshydratation (aux alcools montants), elles subissent un court bain de toluène avant d'être montées entre lame et lamelle, au baume du Canada, en position dorsale, ventrale ou latérale.

Les spécimens utilisés pour les différentes mesures sont montés directement entre lame à cavité et lamelle, dans l'alcool à $70^{\circ}$.

\section{Représentation et nomenclature}

La chétotaxie du corps est représentée selon le schéma fixé par Klein (1964) : une moitié symétrique de segment étalé (fig. $3 C$ ).

La nomenclature des soies céphaliques et corporelles est également empruntée à cet auteur :

- soies céphaliques :

- symbole a dans la rangée antérieure

- symbole p dans la rangée postérieure,

leur numérotation s'effectuant à partir de la ligne médio-dorsale (fig. $1 \mathrm{~B}$ ).

— rangée postérieure des soies corporelles :

- symbole d : soies dorsales (d1 à d3 à compter à partir de la ligne médio-dorsale)

- symbole 1 : soies latérales (11-12 à compter à partir de la ligne médio-dorsale).

- symbole v : soies ventrales (v1-v2 à compter à partir de la ligne médio-dorsale).

La nomenclature des pièces céphaliques est celle retenue par Denis et Bitsch. (in Grassé, 1973).

\section{Description de la larve de $X$. cunicularis}

\section{1 - Troisième stade larvaire}

a - mensurations générales

La longueur totale moyenne est de $3,30 \mathrm{~mm}(3,12$ à 3,56). La tête, mesurée face dorsale depuis le bord antérieur du labre jusqu'au bord postérieur de la capsule céphalique, fait en moyenne $215 \mu \mathrm{m}$ de longueur (210 à 226).

b - chétotaxie et morphologie céphaliques

- La rangée antérieure possède trois soies (a 1 à a 3 ) et la rangée postérieure quatre (p1 à p4) (fig. $1 A$ et B). La longueur moyenne de ces soies est de $9 \mu \mathrm{m}$ (8 à 10) pour a1, $5 \mu \mathrm{m}(4-6)$ pour a2, $6 \mu \mathrm{m}$ pour a, $43 \mu \mathrm{m}$ (25-50) pour p1, $45 \mu \mathrm{m}$ (41-52) pour p2, 
A

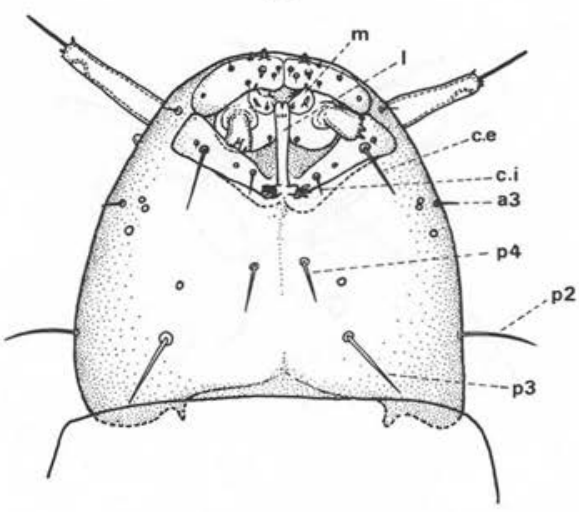

B

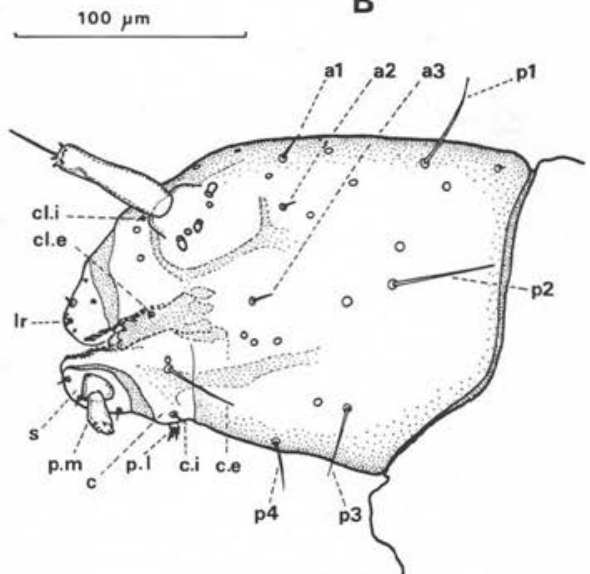

FIg. I. - Larve de X. cunicularis (stade III) - A et B, Tête : A, face ventrale ; B, face latérale gauche.

$a_{1}-a_{3}$ : soies de la rangée antérieure; $p_{1}-p_{4}$ : soies de la rangée postérieure; cl.i., cl.e. : soies clypéales interne et externe ; c.i., c.e. : soies interne et externe du cardo ; c. : cardo ; 1 : labium ; lr. : labrum ; m. : mala ; p.l. : palpe labial ; p.m. : palpe maxillaire ; s. : stipes.

$23 \mu \mathrm{m}(10-32)$ pour p3, $27 \mu \mathrm{m}$ (23-32) pour p4. En arrière de la soie p1 existe une soie petite (inf. à $4 \mu \mathrm{m}$ ) mais constante.

- Le cardo présente deux soies, une interne petite et une externe grande et chez certains exemplaires une minuscule soie accolée à la grande soie externe (fig. $1 \mathrm{~A}$ ). - La maxille présente une soie sur le stipès, et une soie plus deux sensilles sur la mala (fig. 1 A).

- L'ornementation du labre du bord dorsal vers le bord ventral, et du plan médian vers le bord externe est la suivante : une fossette sensorielle médiane impaire ( $\mathrm{fig}$. $2 \mathrm{~B}$ et $C$ ), et de chaque côté :

- une soie au même niveau que la fossette sensorielle,

- une sensille proéminente et une soie très latérale,

- deux minuscules soies et une sensille moins développée que la précédente,

- une soie inféro-externe.

— Le Clypeus possède dans sa partie antérieure deux soies ( fig. 2 C), l'interne étant toujours plus petite que l'externe.

- Enfin une infime soie para-antennaire, très difficile à voir, est inserrée en dedans de l'antenne.

Les mandibules ont une longueur moyenne de $59 \mu \mathrm{m}$ (58 à 60) et elles portent sur le bord antero-dorsal quatre dents arrondies (fig. $2 \mathrm{~A}$ ).

c - chétotaxie du corps (fig. $3 \mathrm{C}$ )

- chétotaxie générale

Pour une moitié symétrique les segments se partagent en 5 types :

- segments thoraciques 1 à $3: 5$ soies dans la rangée antérieure

5 soies dans la rangée postérieure 


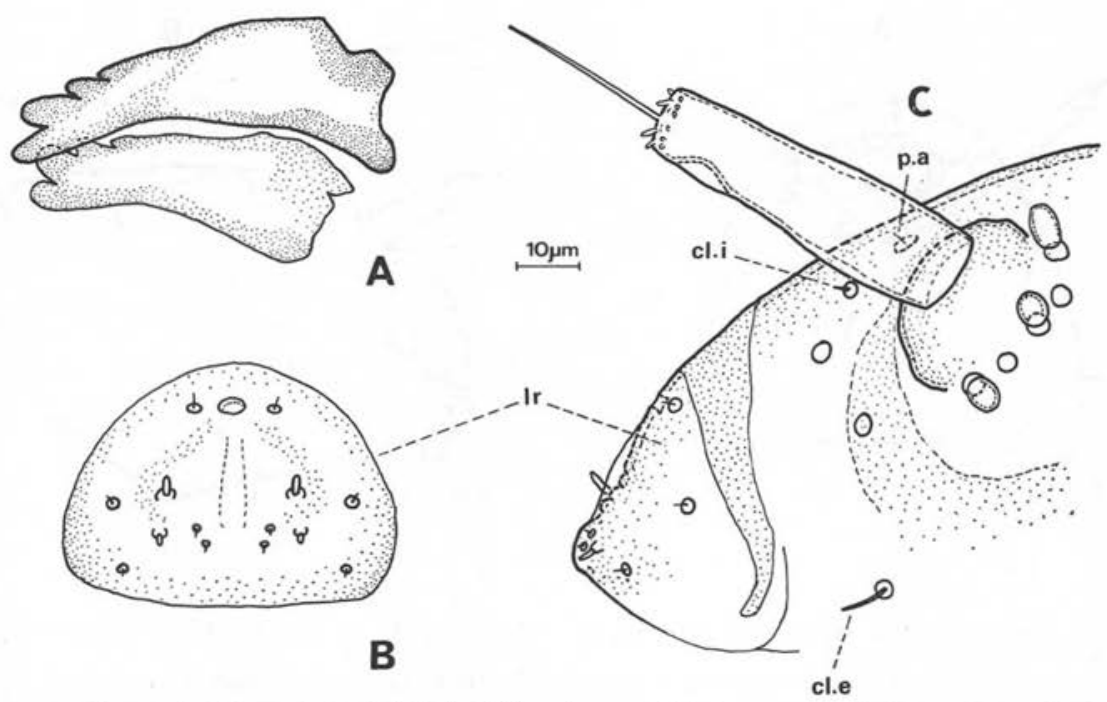

FIG. 2. - Larve de X. cunicularis (stade III) - A : mandibules, vue latérale gauche - B : labre, vue antérieure - C : partie antérieure de la tête, vue latérale gauche ; cl.i., cl.e. : soies clypéales interne et externe; lr. : labrum; p.a. : soie para-antennaire.

— segments abdominaux 1 à $6: 6$ soies dans la rangée antérieure

5 soies dans la rangée postérieure

- segments abdominaux 7 et $8: 6$ soies dans la rangée antérieure

- segment abdominal 9

6 soies dans la rangée postérieure

- segment abdominal 10

: 5 soies dans la rangée antérieure

7 soies dans la rangée postérieure

: 1 soie dans la rangée antérieure

le peigne anal et 3 soies dans la rangée postérieure

les soies para-anales.

Le peigne anal est constitué au total de 16 à 18 soies $(8+8,8+9$ ou $9+9)$ de longueur identique sauf les deux soies médianes, plus courtes et qui s'entrecroisent (fig. $3 \mathrm{~B}$ ).

Les soies para-anales sont insérées à la base des appendices caudaux sur les faces dorso-médiane et internes. Leur nombre est variable (14 à 20 de chaque côté), elles sont de longueurs diverses et ne présentent pas d'arrangement particulier.

- chétotaxie des plaques dorsales

Selon le nombre de soies dans les rangées antérieures et postérieures sur les plaques dorsales, nous pouvons distinguer ( $f i g .3 C$ ) :

$\begin{array}{rr}\text { — segment thoracique } 1 & 3 \text { soies dans la rangée antérieure } \\ & 3 \text { soies dans la rangée postérieure } \\ \text { — segments thoraciques } 2 \text { et } 3: \begin{array}{l}1 \text { soie dans la rangée antérieure } \\ 2 \text { soies dans la rangée postérieure }\end{array}\end{array}$ 

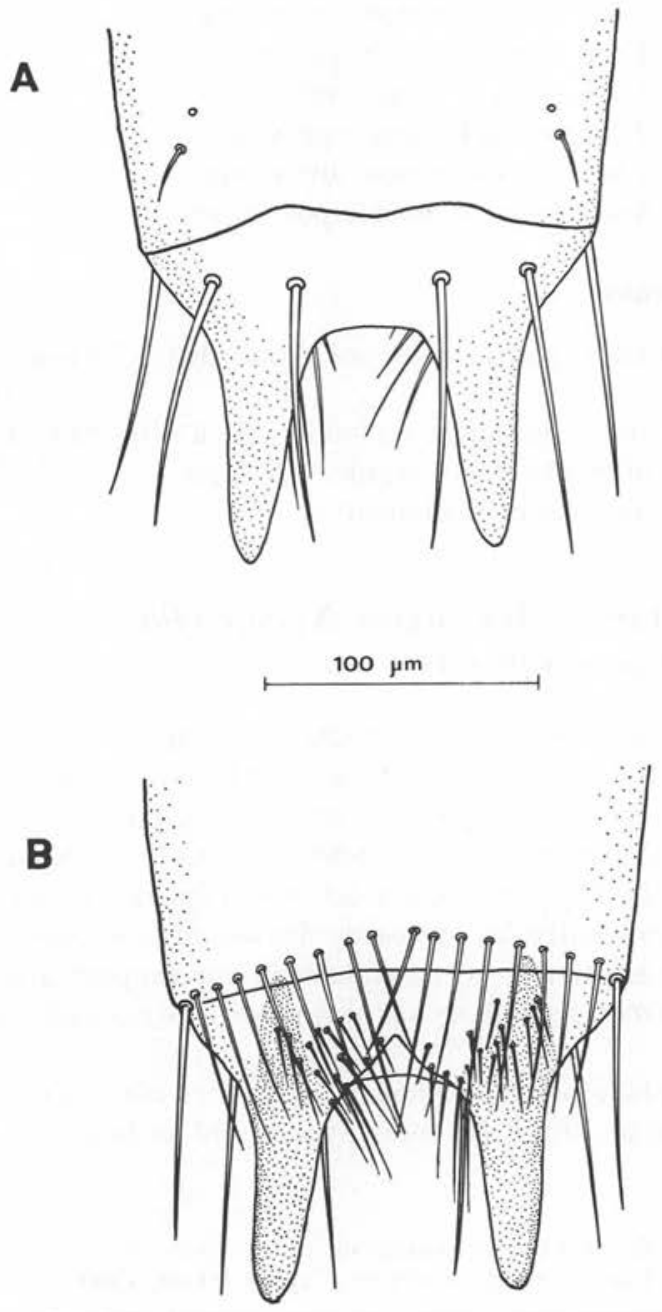

FIg. 3. - Larve de $X$. cunicularis (stade III) - A : segment abdominal ro, face ventrale B : segment abdominal ro, face dorsale - C : schéma de la chétotaxie des segments du corps de la larve (moitié symétrique étalée); p.d. : plaque dorsale. th. 1

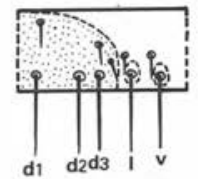

th. 3

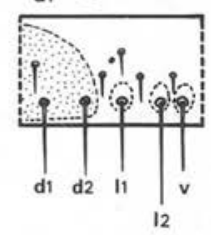

ab.1

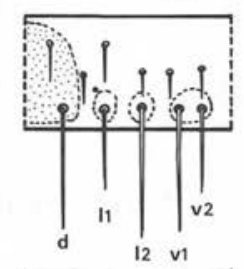

C

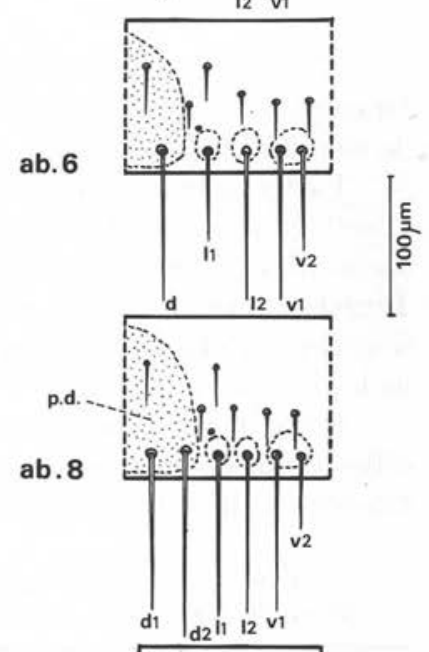

ab. 9

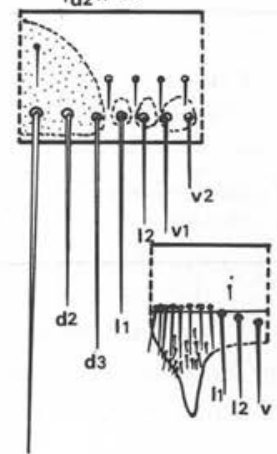


- segments abdominaux 1 à $6: 1$ soie dans la rangée antérieure

1 soie dans la rangée postérieure

- segments abdominaux 7 et $8: 1$ soie dans la rangée antérieure

2 soies dans la rangée postérieure

- segment abdominal 9

: 1 soie dans la rangée antérieure

3 soies dans la rangée postérieure.

\section{2 - Premier et deuxième stade larvaire}

Le stade 1 se reconnaît bien entendu par la présence d'une dent d'éclosion céphaliq' e

La chétotaxie des deux premiers stades larvaires est semblable à celle du troisième stade. Seule la taille sépare ces différents stades (stade 1, lg. moy. : 2,20 mm; stade 2, lg. moy. : 2,60 mm) avec toutefois des recouvrements.

\section{Comparaison avec les larves des autres Xenopsylla du groupe conformis}

$X$. cunicularis est étroitement apparentée aux Xenopsylla du groupe conformis (et en particulier à $X$. ramesis dont elle dérive directement), essentiellement parasites de Gerbillidés, très proches sur le plan morphologique mais aussi écologique.

Parmi celles-ci, seule la larve de $X$. buxtoni a été étudiée en détail, par Klein (1964). Si l'on considère le nombre et la disposition des soies, tant céphaliques que corporelles, aucune différence n'apparaît entre les larves de buxtoni et cunicularis. Toutefois, une minuscule soie insérée en arrière et ventralement par rapport à la soie a 3 est quelquefois présente chez buxtoni; aucun de nos exemplaires de cunicularis ne la possède

D'une façon générale les soies céphaliques de cunicularis sont plus courtes que celles de buxtoni, ainsi chez les larves au stade III nous avons noté les longueurs moyennes suivantes (tableau I) :

TABleau I. - Comparaison de différentes mensurations entre les larves au stade III de $X$. cunicularis et $X$. buxtoni (cette dernière d'après Klein, 1964).

\begin{tabular}{|c|c|c|c|c|c|c|c|c|c|}
\hline \multirow{2}{*}{$\begin{array}{l}\text { longueurs } \\
\text { en } \mu \mathrm{m}\end{array}$} & \multirow{2}{*}{$\begin{array}{l}\text { longueur } \\
\text { moy. totale }\end{array}$} & \multirow{2}{*}{$\begin{array}{l}\text { longueur moy. } \\
\text { de la capsule } \\
\text { céphalique }\end{array}$} & \multicolumn{3}{|c|}{ soies de la r. a. } & \multicolumn{4}{|c|}{ soies de la r. p. } \\
\hline & & & al & a2 & a3 & pl & $\mathrm{p} 2$ & p3 & $\mathrm{p} 4$ \\
\hline $\begin{array}{l}X . \text { cunicularis } \\
X . \text { buxtoni }\end{array}$ & $\begin{array}{l}3300 \\
3420\end{array}$ & $\begin{array}{c}215 \\
175 \text { à } 200\end{array}$ & $\begin{array}{r}9 \\
22\end{array}$ & $\begin{array}{l}5 \\
15\end{array}$ & $\begin{array}{r}6 \\
25\end{array}$ & $\begin{array}{l}43 \\
62\end{array}$ & $\begin{array}{l}45 \\
62\end{array}$ & $\begin{array}{l}23 \\
32\end{array}$ & $\begin{array}{l}27 \\
37\end{array}$ \\
\hline
\end{tabular}

Les mandibules de cunicularis sont plus petites pour un stade donné que celles de buxtoni, pour une longueur céphalique identique : au stade 3,r espectivement $59 \mu \mathrm{m}$ et $68 \mu \mathrm{m}$ en moyenne. D'autre part, les dents du bord interne des mandibules 
sont nettement arrondies et au nombre de 4 chez cunicularis, alors qu'elles semblent pointues chez buxtoni et quelquefois au nombre de 5 .

Kiryakova (1968) indique également 5 dents à la mandibule de Xenopsylla conformis, nuttalli, skrjabini, gerbilli caspica, hirtipes (toutes du groupe conformis). Toutes ces espèces, hormis hirtipes, présentent la même sétation que cunicularis en particulier dans les soies de la rangée postérieure des segments corporels, soit : 5, 5, 5, 5, 5, 5, 5,5, $5,6,6,7 . X$. hirtipes présente par $1 / 2$ segment abdominal une soie supplémentaire soit : $5,5,5,6,6,6,6,6,6,7,7,8$. L'imago de $X$. hirtipes présente également, par rapport aux autres Xenopsylla du groupe conformis une sétation plus abondante.

Toute les larves de ces Xenopsylla du groupe conformis montrent finalement une grande homogénéité morphologique et ne diffèrent que par d'infimes détails (longueur de certaines soies, mandibule).

\section{Comparaison avec la larve de Spilopsyllus cuniculi, autre Pulicidae spécifique du lapin de garenne}

$X$. cunicularis cohabite avec S. cuniculi sur une grande partie de l'aire de répartition de cette dernière. Il semble donc, a priori, intéressant de pouvoir différencier ces larves l'une de l'autre.

La larve de S. cuniculi a été étudiée par Mead-Briggs (1959). Elle se sépare immédiatement de $X$. cunicularis par les caractères suivants :

1 - mandibules : 6 dents chez S. cuniculi, 4 chez X. cunicularis.

2 - chétotaxie céphalique:

a) rangée antérieure : 3 soies (courtes) chez $X$. cunicularis

4 soies (3 longues, 1 courte) chez $S$. cuniculi

b) rangée postérieure : 4 soies chez les deux.

3 - labre

La soie latérale la plus près du bord ventral est remplacée chez $S$. cuniculi par une sensille.

4 - chétotaxie corporelle

sur une moitié symétrique (tableau II) :

TABleau II. - Comparaison de la chétotaxie corporelle chez les larves de $X$. cunicularis et $S$. cuniculi (cette dernière d'après Mead-Briggs, 1959).

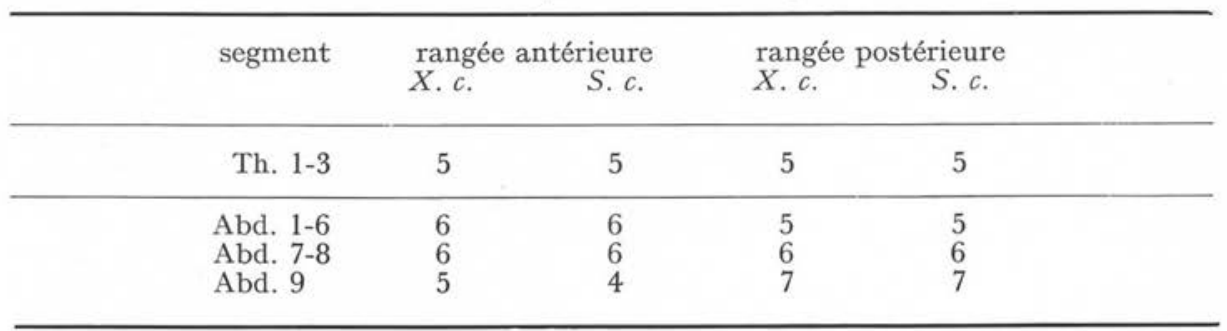


Il convient toutefois de signaler, qu'outre ces critères morphologiques, des critères écologiques permettent de séparer les larves de $X$. cunicularis et $S$. cuniculi dans leur aire de répartition commune :

- les larves de $S$. cuniculi sont présentes uniquement dans les rabouillères.

- les larves de $X$. cunicularis effectuent leur développement dans le sol des galeries des terriers, leur présence restant possible dans les rabouillères mais avec un caractère accidentel.

\section{BIBLIOGRAPHIE}

Beaucournu J. C., Launay H. : Présence en France d'une Xenopsylla selvatique $X$. cunicularis Smit, 1957 (Siphonaptera, Pulicidae) parasite du lapin de garenne. Bull. Soc. Path. exot., 1977, 70, 299-301.

DENIS J. R., Birsch J. : Morphologie de la tête des Insectes. Ordre des Aphaniptères. B - Larves: 552-554. in Grasse P. P., Traité de Zoologie, VIII (1), 799 P. Masson, Paris, I973. KIrJAkova A. N. : (External morphology of Larvae of fleas of the family Pulicidae). Parazitologiya $P, 1968,6,548-558$.

KLEIN J. M. : Contribution à l'étude morphologique externe des larves de puces. Les larves de Xenopsylla buxtoni Jord. 1949, Nosopsyllus iranus iranus Wag. et Arg., I934, et Stenoponia tripectinata irakana Jord., 1958. Bull. Soc. Ent. Fr., 1964, 69, 174-196.

LAUNAY H. : Approche d'une prophylaxie de la myxomatose : écologie des puces du lapin de garenne. Bull. Mens. Off. Nat. Chasse, Déc. 1980, No Sp. Scien. Techn, 213-241.

MEAD-Briggs A. R. : The larva of Spilopsyllus cuniculi (Dale) (Siphonaptera). Proc. R. ent. Soc. Lond. (A), 1959, 43, 27-33. 